


\title{
Systématiser la différenciation raciale à travers le régime juridique de l'indigène : la contribution d'Henry Solus
}

\author{
Isabelle Merle *, Lionel Zevounou **
}

* CREDO, UMR 7308, Aix-Marseille Université, CNRS, EHESS, F-13331 Marseille.

<isabelle.merle@univ-amu.fr>

** Centre de Théorie et Analyse du Droit (CTAD), Université Paris Nanterre, 200 avenue de la République, F-92000 Nanterre.

$<$ lionel.zevounou@parisnanterre.fr>

Q Résumé

Le Traité de la condition des indigènes en droit privé publié en 1927 reste, à ce jour, une œuvre singulière au sein de la doctrine juridique de droit colonial. Il constitue la première et unique tentative de systématisation des rapports de droit privé en droit colonial. Cet article montre de quelle manière la « race» (ancrée ici dans une logique d'altérisation et d'assignation) mobilisée par Henry Solus lui sert à construire une catégorie d'indigène sur laquelle repose la systématisation à laquelle il aboutit.

Droit comparé - Droit international privé-Race-Statut juridique de l'indigène.

Summary Systematizing Racial Differentiation through the Legal Regime of the Indigenous: The Contribution of Henry Solus

Published in 1927, the Traité de la condition des indigènes en droit privé (Treatise on the condition of indigenous people in private law) remains, to this day, a singular work within the legal doctrine of colonial law. It is the first and only attempt to systematize private law relations in colonial law. This article shows how "race" (anchored here in a logic of alteration and assignment) mobilized by Henry Solus helps to construct a category of indigenous on which is grounded the systematization he builds.

Comparative Law - International Private Law - Legal status of the Indigenous - Race. 
Henry Solus est, entre les deux guerres, un juriste reconnu en tant que spécialiste de droit processuel, droit civil et droit commercial dont les principaux travaux ont été plusieurs fois réédités par les éditions Sirey jusque dans les années 1960. Le Traité de la condition des indigènes en droit privé, publié par ces mêmes éditions Sirey en $1927^{1}$, n'a en revanche jamais été réédité. L'ouvrage pourrait apparaître comme une œuvre de jeunesse, singulière et mineure, une sorte d'incursion ponctuelle dans le domaine du droit colonial. Il n'en est rien. Henry Solus fut aussi en son temps spécialiste et praticien reconnu du droit colonial dans lequel il appliqua ses talents de civiliste et pour lequel il soutint un intérêt tout au long de sa carrière. Si Le Traité de la condition des indigènes en droit privé est le seul ouvrage qu'il publie dans ce domaine ${ }^{2}$, Solus enchaîne, en revanche, missions, rapports et enseignements ayant trait à la législation et aux questions coloniales jusqu'à la fin de sa carrière en 1963. Introduit dès les années 1920 par Girault, auteur du classique, Principes de colonisation et législation coloniale publié en 1895, Solus s'inscrit dans cette filiation, représentant avec constance la pensée juridique dominante dans le domaine du droit colonial dont l'une des armes essentielles fut d'élaborer le régime juridique de l'indigène en systématisant la différenciation raciale au sens biologique ou phénotypique ou au sens plus large culturel ou civilisationnel. La race, ancrée dans une évidence "factuelle", devient une catégorie construite grâce au contenu juridique qu'on lui donne alors même qu'elle n'est subsumée par aucune norme supérieure.

À travers l'étude du Traité de la condition des indigènes en droit privé, nous voudrions éclairer ici ce travail de différenciation raciale opéré sur le terrain juridique auquel s'attelle Solus. Il fait miroir en cela à l'ouvrage de Girault, Principes de colonisation et de législation coloniale. En parallèle d'un droit public colonial, sont jetées ainsi les fondations d'un droit privé colonial ${ }^{3}$ afin d'apporter une justification à la différenciation des statuts entre sujets indigènes, citoyens et étrangers. Au fondement de la justification, l'irréductible altérité que les penseurs de la colonisation, en premier lieu les juristes, prêtent aux peuples colonisés, en mobilisant la notion de « race», jamais définie et souvent accolée à d'autres marqueurs de la différence, les mœurs, l'état social ou les civilisations.

\section{Des usages du mot race chez Solus}

\section{I.1. Un usage parcimonieux d'une notion qui reste indéfinie et qui pourtant relève de l'évidence}

Ayant procédé à un travail systématique à partir du Traité de la condition des indigènes en droit privé, nous avons cherché dans le texte à comprendre les usages que Solus fait du mot race, en repérant le nombre d'occurrences et les sujets auxquels

1. Henry Solus, Traité de la condition des indigènes en droit privé. Colonies et pays de protectorat (non compris Afrique du nord) et pays sous mandat, Paris : Éditions Sirey, 1927.

2. Solus écrit de surcroît plusieurs articles et notes d'arrêts publiés aux recueils Dareste et Penant.

3. Structurante dans l'ordre juridique français, la distinction entre un droit public et privé est ici reprise en droit colonial comme une forme de projection du modèle français sur ce dernier sous-système : Pierre LEGENDRE, Trésor historique de l'État en France. L'Administration classique, Paris : Fayard, 1992, p. 167-168. 
celles-ci se rapportent. Plutôt que de traiter de la race en général, il nous a semblé plus utile d'analyser précisément les contextes dans lesquels un juriste tel que Solus juge utile de mobiliser cette notion. Pourquoi ? À quelles fins ? Dans quel sens?

Nous avons repéré dans le texte de Solus 54 occurrences du mot race dans un ouvrage comptant 570 pages, ce qui représente une utilisation relativement limitée. Notons que la race chez Solus ne fait l'objet d'aucune définition, ni même de réflexion spécifique. Le mot incarne pour lui une évidence tenue pour naturelle. Conformément aux canons de son temps, les races et la race existent sans qu'il y ait besoin d'interroger le concept. Il ne s'agit pas d'une catégorie juridique, mais d'un critère socio-anthropologique de sens commun qui participe alors des critères de distinction et de classement des peuples ou civilisations. Solus associe très fréquemment ce critère à d'autres. C'est ainsi qu'il utilise à plusieurs reprises la notion de race suivie de la couleur (de peau), les conditions sociales, l'état social, les mœurs, la religion en concluant par une formule récurrente : «en un mot les différences de civilisation", comme le montre l'exemple suivant.

En instituant une réglementation unique de l'accession des individus à la qualité de français pour toutes les colonies, on méconnait ouvertement la diversité des individus dans lesquels se trouvent les indigènes de notre vaste empire colonial : différences de races, de religion, de mœurs, d'état social, en un mot de civilisation ${ }^{4}$.

Les races ou la race constituent ainsi un marqueur parmi d'autres qui indique parfois une origine géographique ou topographique - la race océanienne, la race aborigène, la race montagnarde (Meo, Lu, Kha) au Laos - ou une spécificité ethnique, phénotypique ou culturelle qui s'ajoute à d'autres spécificités comme les mœurs, l'état social ou la religion :

Les étrangers en colonie ne peuvent être traités de la même manière qu'en France que s'ils sont de race européenne, de race blanche, ou tout au moins dont la civilisation et l'état social correspondent au nôtre. Mais en ce qui concerne certains étrangers qui relèvent d'un État limitrophe de certaines de nos colonies et qui de ce fait, possèdent avec les indigènes de ces colonies une grande affinité de race, de mœurs, d'institutions, de civilisation en un mot, il a paru [...] rationnel de suivre une autre ligne de conduite [les considérer comme des étrangers assimilés aux indigènes] ${ }^{5}$.

L'usage du mot race chez Solus reste relativement confus. À propos de la qualité de Chinois en Indochine, il affirme que le décret organique de 1864 s'est " attaché à discerner la race, l'origine des individus, bien plutôt qu'à considérer leur domicile ou leur lieu de naissance » 6 . On devine là le sens biologique qu'il donne à la race, ce qui est plus clairement explicité dans le traitement qu'il fait du cas des enfants nés de parents inconnus en Indochine. Il y a d'abord l'évidence de «la pure race blanche" pour les enfants nés de parents inconnus, mais "français ou étrangers assimilés ». Il y a ensuite la difficile administration de la preuve par la race pour les enfants métis de parents inconnus afin de les classer du côté des indigènes ou des non indigènes.

4. Henry Solus, Traité de la condition des indigènes en droit privé, p. 122.

5. Ibid., p. 59 .

6. Ibid., p. 65. 
C'est la race qu'il faut considérer pour savoir si l'enfant est indigène ou non indigène [...]. Or la race peut s'établir par tous les moyens de preuve: preuve par écrit, preuve par témoins, preuve par présomption. Ce dernier mode sera surtout, le plus souvent, précieux et fécond. À défaut de toute autre source de renseignement, le tribunal se prononcera d'après l'aspect physique de l'enfant, aspect qui, en général, ne trompe pas. Au besoin, on confiera à une expertise médico-légale, le soin de préciser les caractères ethniques du sujet, de dire s'il est de race indigène, de race française, de race franco-indigène 7 .

Si Solus donne à la race un sens premier lié à la biologie, aux aspects physiques ou phénotypiques, il semble élargir aussi le vocable aux aspects ethniques pour qualifier des peuples spécifiques (les Chams par exemple dont il reconnaît la particularité de la race, des mœurs et des coutumes au Cambodge). Il l'utilise dans un sens encore plus imprécis lorsqu'il l'associe " au tempérament », à la religion, à l'état social, en d'autres termes à un ensemble de marqueurs de distinctions qu'il résume «en un mot» au milieu ou à la civilisation ${ }^{8}$. Dans l'imprécision des qualificatifs, se niche une convocation du racisme scientifique en vogue dans la science anthropologique de l'époque et du racisme tiré du sens commun : celui de l'homme ordinaire pour qui les différences de race et de civilisation relèvent de l'évidence. Le statut de l'indigène se situe quelque part entre celui de citoyen français et d'étranger ${ }^{9}$. Ainsi à propos de la « naturalisation dans le statut ", c'est-à-dire la possibilité pour l'indigène d'accéder à la citoyenneté française tout en gardant son statut personnel, Solus explique :

Tout en permettant à l'indigène d'obtenir la qualité juridique qui consacre son attachement et son dévouement à la France, elle lui fournit le moyen de rester fidèle à ses institutions traditionnelles et de vivre selon les préceptes juridiques qui sont conformes à son tempérament, à sa race, à sa religion, en un mot au milieu dans lequel il est accoutumé à déployer son activité 10 .

Mais c'est aussitôt pour mieux démentir l'affirmation en dressant la liste des « réels dangers » que présente l'accession « en masse » ou sur demande individuelle des indigènes aux droits et obligations de la citoyenneté française et en particulier à la jouissance des droits politiques français jointe au maintien du statut personnel, alors même que ceux-ci relèvent de civilisations et d'organisations sociales profondément différentes.

Il peut être excessif et déraisonnable de vouloir soumettre en même temps un indigène à des institutions de droit public d'un côté et à des institutions de droit privé de l'autre, qui les unes et les autres correspondent à des civilisations et à des organisations sociales profondément différentes. C'est méconnaître gravement les rapports qui enchaînent la loi avec le milieu qu'elle est destinée à régir ${ }^{11}$.

7. Ibid., p. 84 .

8. On retrouve un mode de raisonnement similaire durant la même période s'agissant de l'interprétation de la race par la Cour suprême des États-Unis et de l'acquisition de la nationalité: United State v. Bhagat Singh Thind, 261 US, 204 (1923) ; Ozawa v. United States, 260 US, 178 (1927). Sur l'analyse de ces deux cas: Ian Haney LoPEZ, White By Law. The Legal Construction of Race, New York: New York University Press, 2006, spéc. chap. 4, p. 56-77.

9. Yerri URBAn, L'indigène dans le droit colonial français 1865-1955, Paris: LGDJ, Fondation Varenne, 2010, p. 441-443. Solus reprend ici le raisonnement d'un arrêt rendu par la cour d'appel d'Indochine le 27 octobre 1911 cité par Y. Urban, p. 443.

10. Ibid., p. 129.

11. Ibid., p. 135. 
Parmi les caractéristiques du «milieu », il y a la race perçue comme l'un des déterminants, parmi d'autres, du degré de civilisation des peuples et de leurs aptitudes à participer pleinement à la cité française. Pour Solus, il est un principe auquel il ne dérogera jamais, l'intime conviction que «les différences de races, de religion de mœurs, d'état social, en un mot de degré de civilisation » imposent de "renoncer à l'unité mystique des êtres humains qui pourraient recevoir indifféremment les mêmes lois et s'y adapter » 12 : c'est là un principe partagé par l'ensemble de la doctrine coloniale de l'époque. Qu'il s'agisse de la naturalisation dans le statut ou pire encore, de son point de vue, d'une réglementation unique de l'accession des indigènes à la qualité de citoyen français pour toutes les colonies, il y aurait là un danger profond d'aveuglement face à ce qui l'obsède au fond : la différence.

\section{I.2. La race au service d'un projet différentialiste}

Solus propose la définition suivante de l'indigène sujet français que peu d'auteurs, nous dit-il, ont tenté jusqu'ici de systématiser :

On entend par indigènes sujets français les indigènes qui, originaires des colonies faisant, par suite de l'annexion, partie intégrante du territoire français, sont soumis à la souveraineté directe de la France, mais ne possèdent pas les droits de citoyens 13 .

Nul besoin de mobiliser la race pour traiter du clivage qui sépare les habitants des colonies, français par nationalité, mais distribués de part et d'autre d'une frontière avec, d'un côté ceux qui ont les droits de citoyens et de l'autre, ceux qui ne les ont pas. Les indigènes sujets français ne jouissent pas des droits politiques et possèdent un statut personnel spécial «dont les droits dérivent des lois et des coutumes indigènes locales que la France a maintenues en vigueur lors de l'annexion, sous certaines conditions qui varient d'ailleurs de colonie à colonie»14. Ainsi se dessine « une situation intermédiaire entre les citoyens français et les étrangers " 15 .

Solus engage une réflexion plus précise, lorsque contestant «la théorie de l'indigène citoyen français [qui] a eu autrefois son heure de grande vogue »16, il critique l'accès ouvert à la pleine citoyenneté en certains territoires et en certaines circonstances historiques à des hommes de couleur dans les anciennes colonies ou à des indigènes. La loi de 1833 qui accorda la complète égalité de traitement entre les hommes de "race blanche» et les hommes de couleur libres, en Martinique, en Guadeloupe, à la Réunion et en Guyane, témoigne, selon lui, d'une «ignorance profonde, mais excusable alors, des questions coloniales » 17 issue d'une utopie résultant des idées de liberté, d'égalité et de fraternité chères à la Révolution. Par extension ou dans des circonstances historiques particulières, l'accès à la pleine citoyenneté française fut accordé aux indigènes de Sainte-Marie de Madagascar, aux natifs des quatre communes du Sénégal et aux habitants du Royaume de Pomaré à Tahiti. Tous ces

\footnotetext{
12. Ibid., p. 122.

13. Ibid., p. 35.

14. Ibid., p. 37.

15. Ibid.

16. Ibid., p. 15.

17. Ibid., p. 17.
} 
cas sont pour Solus critiquables au sens où « les indigènes étaient le plus souvent attachés à leur statut personnel propre; ils vivaient dans un état économique et social très différent de celui auquel correspond la loi française. Et c'était une utopie de croire qu'une formule légale avait le pouvoir de les transformer hic et nunc en citoyens français aptes à jouir de tous les droits civils et politiques français» 18 . Cependant, il admet que l'histoire s'impose à lui en ce qui concerne le devenir des hommes de couleur et des affranchis issus de l'esclavage qui, sans contestation, sont désormais citoyens français ${ }^{19}$. La question du maintien ou non du statut personnel est cruciale dans les polémiques qui ont eu lieu à propos des habitants de Sainte-Marie de Madagascar, les natifs des quatre communes du Sénégal ou encore les descendants du Royaume de Pomaré. Solus s'attache à montrer les incertitudes à ce sujet et juge très contestables en particulier les solutions juridiques adoptées par la loi du 29 septembre 1916 pour les natifs des quatre communes du Sénégal, votée « avec une hâte regrettable " ${ }^{20}$. Dans le principe, hormis ces cas d'exception, reconnaître aux indigènes la qualité de Français revient, pour Solus, à «ne tenir aucun compte des différences de races et de couleur, de conditions sociales, de mœurs et de religion, en un mot des différences de civilisation qui séparent les Français d'origine et les indigènes ${ }^{21}$.

La race est mobilisée au service de ce différentialisme, avec d'autres déterminants qui servent à construire une frontière entre indigènes et citoyens qu'il voudrait très difficilement franchissable. Solus ne fait preuve d'aucune ouverture lorsqu'il s'agit de penser une réglementation unique de l'accession des indigènes à la qualité de citoyen français à la fin des années 1920, pas plus qu'il ne défend le projet de citoyenneté dans le statut qui commence à être réfléchi et sera soutenu par le ministre Maurice Violette en 1936. Il souligne à plusieurs reprises les différences pour interroger "les aptitudes" des indigènes en rappelant la diversité des degrés de civilisation selon les peuples et craint, par-dessus tout, "l'entrée en masse d'une foule d'indigènes dans le cadre de la cité française » ${ }^{22}$. Ce n'est pas un bon moyen, selon lui, de reconnaître leur loyauté et l'impôt du sang que beaucoup ont payé sur les champs de bataille de la Grande Guerre.

Les indigènes en retour de leur attachement et de leur service ont toujours trouvé de notre part, effort persévérant en vue d'assurer la paix et la sécurité des territoires sur lesquels ils vivent, le respect de leurs coutumes et de leurs croyances, la conservation de leur race, leur développement intellectuel, l'amélioration des conditions économiques, matérielles et morales de leur existence 23 .

La «conservation de la race» vient ici en appui d'une démonstration essentialiste qui vante les mérites des traditions, car «l'expérience a souvent prouvé que

\footnotetext{
18. Ibid.

20. Ibid., p. 25.

21. Ibid., p. 14-15.

22. Ibid., p. 122.

23. Ibid., p. 125.
}

19. Quoiqu'on ne puisse pas revenir dessus, Solus considère l'intégration des anciens esclaves dans la citoyenneté française comme une erreur historique. 
l'indigène le plus dévoué à notre cause est celui qui est resté fidèle à ses mœurs, à ses traditions familiales, à sa religion [et Solus aurait pu ajouter à sa race] qui n'a cure de renoncer à tout ce patrimoine pour devenir citoyen français » 24 .

Pour les plus "évolués " d'entre eux, mieux vaudrait «qu'on augmente leurs droits politiques dans la cité indigène [...], domaine qui les intéresse le plus et qui est sans aucun doute, celui de leurs intérêts particuliers " 25 . Chacun chez soi dans sa race, ses coutumes, ses lois civiles et la colonie ne s'en portera que mieux. Telle est la devise de Solus.

Par-delà les présupposés essentialistes dont elle fait l'objet, l'invocation de la race chez Solus sert plusieurs usages. Dans certains cas, la différence de race sert à construire une factualité totalement artificielle. Cette factualité renvoie de manière allusive aux savoirs anthropologiques de l'époque sur la hiérarchie des civilisations et de races. Par cet artifice tenant lieu de fait, la construction de la catégorie d'indigène apparaît naturellement correspondre à des " milieux ", " sociétés ", " civilisations ». Il est dès lors possible d'imputer à l'indigène - et en creux, au régime juridique qui lui est attaché - une volonté fictionnelle d'auto-assignation. Celle-ci ne cherche pas à l'extraire du contexte social dans lequel il évolue s'agissant de son statut personnel. Au fond, le raisonnement de Solus auto-assigne l'indigène en lui imputant une volonté globale de satisfaction de sa propre condition, tant sociale, raciale que de genre ${ }^{26}$. Plutôt que de recourir à un raisonnement juridique de type classique, c'est-à-dire autoréférentiel (en comparant les normes réputées inférieures avec les normes supérieures), la catégorie d'indigène et plus largement le droit colonial procèdent par un raisonnement inverse: l'artificialité des faits (civilisations, culture, coutumes, etc.) tient lieu d'argument d'autorité permettant de construire un régime juridique érigé en frontière d'assignation raciale par la même occasion.

\section{Par quel raisonnement Solus parvient-il à systématiser cette différenciation?}

Une fois cette prémisse posée, encore faut-il pouvoir résoudre les éventuels conflits qui peuvent naître entre les «coutumes » du colonisé et le droit du colonisateur. Le rapport de conflit entre ces deux types de systèmes normatifs demeure inégal au profit du second conformément au rapport de force colonial et à l'assujettissement

\footnotetext{
24. Ibid., p. 126.

25. Ibid., p. 125.
}

26. Un exemple typique de cet argument tient dans un arrêt rendu par la Cour de cassation en 1933. La Cour de cassation et avant elle la cour d'appel de Nîmes, s'opposent, dans cette affaire, à la reconnaissance d'un mariage d'un administrateur colonial, en Guinée Conakry, avec deux épouses indigènes selon la coutume: Cass., 14 mars 1933, Consorts de Cousin de Lavallière c. Époux de la Bernardie, Sirey, 1, 1934, p. 161 ; CA Nîmes, 17 juin 1929, Consorts de Cousin de Lavallière c. Époux de la Bernardie, Sirey, 2, 1929, p. 129. Dans l'arrêt d'appel commenté par Henry Solus, la Cour mobilise le travail doctrinal de ce dernier afin de justifier la nullité du mariage à la mort de cet administrateur et suite à l'action introduite par les enfants issus de cette union visant à faire reconnaître leur légitimité et prétendre à la succession. La Cour de cassation rejette le recours ; elle va même encore plus loin, dans la mesure où elle ne reconnaît même pas à l'union le caractère de mariage putatif (autrement dit, un mariage conclu de bonne foi). On a ici une illustration frappante de la manière dont l'interprétation par les juges de la catégorie d'indigène rendue à travers le prisme de la différenciation raciale sert de garde-fou aux tentatives européennes de « décivilisation " (il faut entendre par-là, l'adoption par des Européens des coutumes indigènes). 
de peuples et territoires coloniaux à la puissance conquérante. Solus s'emploie à le démontrer juridiquement. Ce qui est intéressant dans cette démonstration, c'est précisément que la primauté du droit du colonisateur sur le droit "coutumier » repose sur l'évidence faussement factuelle déjà évoquée, d'auto-assignation de l'indigène. En partant de cette prémisse, Solus peut alors proposer une systématisation dans laquelle les conflits entre droit du colonisateur et droit coutumier devraient naturellement déroger aux conflits ordinaires que connaît le droit international privé naissant. Dans la systématisation proposée par Solus, les conflits entre le droit du colonisateur et les "coutumes " indigènes ne relèvent pas de conflits interprovinciaux ou de conflits liés à une annexion. Un détour par les auteurs qui traitent de ces deux dernières questions apparaît nécessaire, afin de mieux saisir la part d'assignation raciale sous-tendue par la catégorie d'indigène.

En effet, considérer qu'il existe des mœurs et des « coutumes » préexistantes au droit du colonisateur, c'est encore admettre qu'un « conflit de lois » puisse s'élever entre les deux systèmes. Précisons que le " conflit de loi » dont nous parlons ici est celui qui prévaut usuellement dans les rapports internationaux de droit privé 27 , entre États souverains : or une telle hypothèse n'est tout simplement pas admissible en contexte colonial, au risque de ruiner le principe de la souveraineté de l'État français. Reste une seconde hypothèse, moins radicale : celle d'un potentiel conflit de statuts - précisons : conflit de statuts en droit interne - sur lequel il y a lieu de s'attarder plus longuement. Le conflit de statuts met aux prises le droit national avec une ou plusieurs coutumes locales. Ce cas de figure n'oppose pas deux États souverains ; il oppose, au sein d'un même système juridique, deux normes issues de deux "sources" différentes. Le cas typique que connaît bien Solus est celui des rapports entre le droit alsacien mosellan et les lois françaises. Qualifié de conflit interprovincial ${ }^{28}$, il est abordé par Jean-Paulin Niboyet dès $1925^{29}$. Entre 1870 et 1918, le droit allemand s'est superposé au Code civil de 1804 en vigueur dans le territoire alsacien mosellan. Après la Première Guerre mondiale, tant le régime que les conflits éventuels résultant de la confrontation entre la loi française en vigueur dans le reste de la France et le droit alsacien mosellan sont régis par une loi adoptée le 24 juillet 1921 ; mais, parce que cette dernière loi ne se prononce pas toujours clairement sur les juridictions compétentes chargées de régler les litiges qui en sont issus, l'ouvrage de Niboyet entend y remédier d'un point de vue doctrinal. Dans l'hypothèse d'un conflit de loi entre le droit alsacien mosellan et le droit national, le conflit se résout par référence à la loi où le conflit à son siège ${ }^{30}$. Niboyet, spécialiste de droit international, dresse un parallèle avec les conflits de

27. Le conflit de loi met classiquement aux prises un conflit de souverainetés. Le concept ne peut, s'agissant du droit privé colonial, être utilisé, car cela reviendrait à reconnaître aux territoires colonisés une souveraineté impossible.

28. Rappelons que cette thèse des conflits interprovinciaux que la doctrine systématise est défendue par des auteurs en France comme Antoine Mailher de Chassat, Charles Aubry, Charles-Frédéric Rau, Armand Lainé, Antoine Pillet, Jean-Paulin Niboyet, Léon Julliot de la Morandière entre autres.

29. Jean-Paulin Niвоует, Conflits entre les lois françaises et les lois locales d'Alsace et de Lorraine en droit privé. Commentaire de la loi du 24 juillet 1921, Paris : Libraire de la Sté du Recueil Sirey, 1922.

30. Ibid., p. 108. 
type colonial qui, dans la logique du droit international, devraient être classés dans les conflits interprovinciaux. Cependant, Niboyet se rend bien compte que cette classification pose problème si elle permet de mettre sur le même plan les conflits alsaciens mosellans et coloniaux. Le conflit alsacien mosellan met aux prises des régimes de droit « civilisés » qui ne sauraient être réduits à ce que la doctrine internationaliste se représente comme des régimes primitifs, dans le cas des conflits coloniaux ${ }^{31}$.

Si l'on essaye d'en déconstruire le raisonnement, la distinction proposée par Niboyet entre le conflit de type colonial et le conflit alsacien mosellan repose sur une gradation des régimes de droit allant des colonisés (que l'on rapproche de la figure du barbare en droit romain) aux sujets alsaciens mosellans classés parmi les civilisés. On retrouve ici, peu ou prou, le même présupposé développé par Solus, à savoir que les «coutumes » en contexte colonial demeurent nécessairement tributaires du stade d'évolution - en l'occurrence primitive - des civilisations dont elles sont issues. De fait, il ne peut être question de mettre sur un pied d'égalité les conflits alsaciens mosellans et les conflits de type colonial. Les premiers sont réglés par une loi et obéissent à ce titre à la rationalité du droit international privé émergeant : respect des droits acquis, conflits de qualification, etc. Les seconds, qu'il s'agisse du droit musulman et des coutumes «indigènes ", s'appuient sur des catégories et un régime qui relèvent du statut personnel. En cas de conflit entre la coutume indigène et le droit français, c'est le second qui, par principe, prévaut sur le premier.

La thèse de Michel Eliesco ${ }^{32}$ se consacre spécifiquement aux conflits d'annexion, c'est-à-dire des conflits entre lois au sein d'un État souverain, entre le droit de la puissance colonisatrice et le droit des peuples colonisés. Elle constitue une autre manière de considérer le conflit colonial et d'intégrer le droit colonial dans le droit international privé.

Eliesco rejette par exemple, après une étude minutieuse des conflits de lois en Algérie, l'analogie que ses collègues font entre lois «barbares » en droit romain et coutumes "indigènes " afin de disqualifier ces dernières pour les exclure du droit civilisé. Il remarque que lorsqu'il est question de régir les biens, qu'ils soient meubles ou immeubles, le statut disparaît au profit de l'application du droit civil en vigueur dans la colonie. Le récit considérant le statut personnel indigène comme un principe général par lequel s'applique la loi coutumière - y compris concernant les transactions des biens que Solus et Niboyet affirment - trouve ici une exception notable 33 . La puissance coloniale sait habilement faire disparaitre le régime du statut personnel en matière de biens. Outre ces questions de transactions immobilières, Eliesco met aussi en lumière l'usage contradictoire de l'ordre public colonial : s'il connaît des limites en droit international privé, l'ordre public en droit colonial n'en connaît aucune ${ }^{34}$.

31. Ibid., p. 106.

32. Michel ELIESCO, Essai sur les conflits de loi dans l'espace, sans conflit de souveraineté: les conflits d'annexion, Paris : Picart, 1925. Voy. aussi : Judith SURKIS, «Propriété, polygamie et statut personnel dans l'Algérie coloniale, 1830-1973 ", Revue d'histoire du XIX siècle, 41 (2), 2010, p. 27-48.

33. Michel Eliesco, Essai sur les conflits de loi dans l'espace, sans conflit de souveraineté: les conflits d'annexion, op. cit., p. 246-248.

34. Ibid., p. 335. 
On voit combien l'analyse d'Eliesco met à nu l'incohérence de la systématisation proposée par Solus. Et c'est bien parce qu'il recherche une telle cohérence à partir d'un raisonnement autoréférentiel, qu'il propose d'en revenir, comme certains juristes du premier Empire colonial, à la disparition progressive des «coutumes » indigènes au profit du droit métropolitain et donc à une assimilation juridique complète, contrairement à la lecture d'un juriste comme Solus. Alors que celui-ci explique la cohérence du régime juridique appliqué à l'indigène en dehors du droit - en s'appuyant sur une factualité artificielle -, le travail d'Eliesco met en lumière les faiblesses d'une telle systématisation en recourant uniquement à des explications normatives. Solus, qui a bien perçu cette difficulté, écarte le travail d'Eliesco d'un revers de main. Il le résume de manière caricaturale en ces termes: « les solutions, d'ailleurs fragmentaires qu'il propose relativement à ce dernier [Solus parle ici de la manière dont la thèse d'Eliesco aborde le conflit colonial], ne peuvent pour la plupart, être retenues, faute d'une connaissance suffisante de l'ensemble et des détails de la matière » 35 . Solus ne peut, en toute logique, conclure qu'à une différence de nature entre conflit colonial et conflit de droit international privé. De cette différence de nature, Solus en déduit une différence de régime, implicitement fondée sur une altérité raciale « factuelle » 36 . La frontière qu'il érige doit être très difficilement franchissable comme le montre la critique féroce qu'il formule à l'encontre de la loi dite «Diagne " garantissant la citoyenneté à tous les natifs des «Quatre Communes " du Sénégal, votée le 29 septembre 1916, qui en constitue un exemple topique. Solus exprime ses craintes que le statut de citoyen soit accordé à des populations aux «mœurs » différentes de celles de la civilisation occidentale; le risque d'un potentiel conflit de lois entre le droit métropolitain et le droit musulman est aussi évoqué comme risque de perturbation des modes de vie antérieurs; enfin, la loi de 1916 est vue par Solus comme une loi de pure « opportunité » faisant désordre 37.

\section{Y a-t-il des exceptions selon Solus ?}

Si l'on met en perspective le travail de Solus sur le droit colonial, on prend la mesure d'un différentialisme à multiples facettes ou, si l'on préfère, de ce que l'assignation raciale sous-jacente à ses écrits prend des contours très différents selon les contextes. Solus plaide par exemple, dans la revue Renseignements coloniaux, pour un statut différencié des indigènes convertis à la foi chrétienne. Sans les intégrer pleinement au sein du régime du Code civil, il soutient que: "soumettre à l'organisation de la famille, le mariage, ses conditions de validité et ses effets, à l'application du Code civil » 38 permettrait de prendre acte de l'évolution de

35. Traité de la condition des indigènes en droit privé, op. cit., p. 7.

36. Ce point transparaît lorsque Solus s'essaie à définir l'indigène : «reconnaître aux indigènes la qualité de citoyens français, c'est, ainsi que nous venons de l'établir, leur accorder la jouissance de tous les droits civils et politiques qui appartiennent aux nationaux français d'origine dans la colonie. C'est donc ne tenir aucun compte des différences de race et de couleur, de condition sociale, de mœurs et de religion, en un mot des différences de civilisation qui séparent les français d'origine et les indigènes » in Traité de la condition des indigènes en droit privé, p. 14-15.

37. Ibid., p. 26-33.

38. ID., « Le statut juridique des indigènes chrétiens ", Renseignements coloniaux, 7, 1935, p. 120. 
cette catégorie d'indigènes. L'assimilation culturelle, par le moyen de la religion en l'occurrence, permet de déroger de manière restrictive à l'auto-assignation supposée de l'indigène. Par contraste, sollicité en mars 1941 par le ministère des Colonies, Solus exige d'appliquer sur le territoire métropolitain le principe de respect des institutions indigènes lorsqu'il est question de restreindre aux travailleurs « annamites » l'accès à la nationalité métropolitaine.

On lui demande de rédiger une consultation afin de mettre fin aux entreprises de mariages qui ont lieu entre la main-d'œuvre indochinoise, venue, dès 1939, prêter main-forte à l'industrie de guerre ${ }^{39}$ et des ressortissantes françaises. La possible généralisation des mariages mixtes inquiète le ministère du Travail de Vichy. Puisqu'il n'est pas possible d'invoquer une norme nationale interdisant de telles unions, on demande à Solus de proposer des arguments conduisant, dans les faits, à rendre impossible ces mariages mixtes. Solus s'y emploie, de deux manières. Il conseille à l'administration "d'informer " les ressortissantes françaises qui souhaitent se marier avec des travailleurs " annamites» que l'homme indochinois " est soumis à la loi personnelle admettant la polygamie » 40 .

Quant aux travailleurs " annamites " - dont on rappelle qu'ils vivaient dans des camps et étaient soumis à une discipline militaire stricte -, Solus préconise à l'autorité administrative, en l'occurrence le ministère du Travail, d'appliquer rigoureusement, sur le territoire métropolitain, les obligations exigées par la loi personnelle du demandeur dans sa colonie afin d'entraver sa demande en mariage (vérification de l'âge requis, consentement exigé par la loi personnelle, vérifier que le demandeur ne soit pas engagé dans les liens d'un précédent mariage non dissous). Il précise, qui plus est, que le commandant de légion - titre attribué au responsable des campements où étaient stationnés les travailleurs indochinois - peut faire obstacle à la demande de mariage formulée par un travailleur "annamite ». On voit par-là que Solus réintroduit sur le sol métropolitain les prescriptions du droit colonial : «l'annamite» qui souhaite se marier avec une Française est soumis à sa " loi personnelle", celle du régime indigène par lequel il est régi sur son territoire d'origine. Pour les Françaises qui souhaitent se marier malgré tout, la figure de "l'annamite " polygame, violent et fourbe, tirée du jugement rendu par le tribunal civil de Hanoï est utilisée comme moyen de dissuasion ${ }^{41}$. Par son travail de légiste, Solus contribue à perpétuer, sur le territoire métropolitain, des catégories et raisonnements juridiques qui participent à instaurer des frontières raciales entre les peuples qui composent l'Empire. La religion, les "coutumes et les mœurs", "l'histoire", servent comme autant de ressources argumentatives pour maintenir ou assouplir selon les besoins, le principe du respect des coutumes indigènes. Aussi, le différentialisme à la source du raisonnement qu'il propose n'est-il pas figé

39. Décret du 29 novembre 1939.

40. Solus convoque ici un jugement du tribunal civil de première instance de Hanoï où le divorce fut prononcé, car le mari avait caché à sa femme française qu'il vivait avec six concubines : Tribunal civil de $1^{\text {re }}$ instance de Hanoï, 10 mars 1929. Dossier "Consultation » sur la question des mariages mixtes entre travailleurs « annamites » et ressortissantes françaises, 24 mars 1941, ANOM, 1ECOL 134, p. 3.

41. Ibid. 
quoique très rigide lorsqu'il s'agit de reconnaître des mélanges (métissage) ou encore valider un accès «brutal » à la citoyenneté française. Cette ligne, Solus n’y dérogera pas, y compris lorsque la Quatrième République cherchera à rompre avec les régimes coloniaux dérogatoires ${ }^{42}$. Consulté comme spécialiste de la commission constitutionnelle de la première constituante présidée par Pierre Lampué, Solus va encore s'employer à combattre l'extension d'une citoyenneté pleine et entière à l'ensemble des ressortissants de l'Union française privilégiant rigoureusement une citoyenneté différenciée et dégradée.

La race selon Solus ne se limite pas à des critères biologiques ou phénotypiques, mais renvoie à une catégorie construite plus large englobant des critères culturels, sociaux et civilisationnels. S'il utilise le terme avec parcimonie, celui-ci lui est essentiel pour définir, interpréter et comprendre le statut de l'indigène et la cohérence du régime juridique qu'il lui échafaude. De ce point de vue, l'ouvrage de Solus apporte une cohérence à l'incohérence intrinsèque du système de domination coloniale. Rappelons que le Traité de la condition des indigènes répond à la commande de Girault et plus largement des milieux coloniaux du moment. L'ouvrage, par la lecture différentialiste systématique qu'il propose, structure de façon essentielle le droit colonial tel qu'il se déploie jusqu'en 1946.

L'analyse critique du travail de Solus ouvre aussi de nouvelles perspectives de recherche quant à la genèse du droit international contemporain d'une part et du droit comparé de l'autre. Est-on jamais sorti, après les indépendances, des préjugés opposant droit coutumier et "droit commun " autrement dit droit de l'ancienne métropole? Les uns exaltant les coutumes au nom de l'authenticité (et souvent ses aspects les plus conservateurs), les autres exaltant, au contraire, l'assimilation parfaite et donc l'application du modèle métropolitain ${ }^{43}$. Le passage du droit colonial au droit d'outre-mer puis aux droits africains interroge les héritages coloniaux liés à la manière dont ces systèmes furent conceptuellement pensés. L'acculturation et la formation des juristes originaires des anciennes colonies, en particulier dans les pays africains, constituent sur ce point un exemple topique. En Nouvelle-Calédonie, le maintien du "statut particulier " pour les Kanak dans le domaine du droit civil, les relations asymétriques entre «droit particulier» et «droit commun » tel qu'on le pensait sous la Quatrième République et le rééquilibrage récent de ce que l'on appelle désormais «le droit coutumier» sont aussi à réfléchir sous le prisme de ces héritages. Dans la persistance des tensions et des confrontations entre deux modèles juridiques que l'on voulait opposer se nichent les continuités d'un ordre politico-juridique élaboré sous la période coloniale et auquel l'ouvrage de Solus a grandement contribué. Au cœur de cet ordre politico-juridique, la race, qui doit être comprise aujourd'hui en tant que catégorie socialement construite et utilisée comme un concept heuristique qui permet d'apporter la complexité nécessaire à la lecture et la compréhension du droit contemporain et de ses héritages.

42. Frederick COOPER, Français et Africains ? Etre citoyen au temps de la décolonisation, Paris : Payot, trad. C. Jeanmougin, 2014.

43. Voir le travail désormais classique sur cette question de : Mahamood MAMDANI, Citizens and Subjects: Contemporary Africa and the Legacy of Late Colonialism, Princeton : Princeton University Press, 1996. 


\section{- Les auteur-rices}

Isabelle Merle est directrice de recherche au CNRS (Centre de Recherche et de Documentation sur l'Océanie), historienne de la colonisation, spécialiste de l'histoire du Pacifique et plus particulièrement de la Nouvelle-Calédonie. Elle s'est également intéressée à la réédition de récits de voyage du XVIII ${ }^{e}$ siècle et est l'autrice de plusieurs articles traitant des questions coloniales et post-coloniales. Elle a notamment publié :

- Expériences coloniales. La Nouvelle-Calédonie. 1853-1920, Toulouse: Anacharsis, 2020 [Belin, 1995] ;-L'indigénat. Genèses dans l'empire français. Pratiques en NouvelleCalédonie (avec Adrian MUCKLE), Paris : CNRS éditions, 2019;

- Watkin TENCH, Expédition à Botany Bay. La fondation de l'Australie coloniale (introduction et notes d'Isabelle MERLE), Toulouse : Anacharsis, 2020 [2006].

Lionel Zevounou est juriste, maître de conférences en droit public à l'Université Paris Nanterre, chercheur au Centre de théorie et Analyse du droit (CTAD, UMR 7074). Il est membre junior de l'Institut universitaire de France. Parmi ses publications :

- Race et droit (dir.), Paris : Institut francophone pour la Justice et la Démocratie, coll. «Transition et justice», 31, 2021. 


\section{Éditorial}

\section{Dossier}

Penser la race en juriste : lectures critiques coordonné par Silvia Falconieri, Laetitia Guerlain et Lionel Zevounou

Silvia Falconieri, Laetitia Guerlain, Lionel Zevounou

Laetitia Guerlain

Claude Blanckaert

Isabelle Merle, Lionel Zevounou

Silvia Falconieri

Paul-André Rosental

Guillaume Richard

Guillaume Mouralis

Documents à l'appui
Les juristes et la race. Analyse critique à partir de quelques textes (1880-1930). Présentation du dossier

Refonder le droit sur la race : la philosophie juridique d'Edmond Picard

Une culture d'imprégnation. Genèse et apories de la notion de « droit racial » chez Edmond Picard

Systématiser la différenciation raciale à travers le régime juridique de l'indigène : la contribution d'Henry Solus

"Race », santé et " génie français ». Un commentaire de Paul Esmein du Code de la famille de 1939

La race, quelle race ? Significations et performativité de la "protection de la race " dans le Code de la famille de 1939

Roger Bonnard et la race en droit nazi

Entre lecture empathique et stratégie de distinction. Le racisme national-socialiste selon Roger Bonnard

\section{Droit et Société au Japon}

Gakuto Takamura

Adrienne Sala, Eri Kasagi

Makiko Shimizu

Yuki Asano

Simon Serverin
La « conscience juridique » aux États-Unis : réceptions comparées en France et au Japon

Judiciarisation de la mort et du suicide par surmenage et cause lawyering à la japonaise?

Réalité et transformation des "pratiques de relations commerciales japonaises » : le cas de l'architecture modulaire

Le raisonnement juridique pluraliste

La légitimité religieuse du pouvoir dans l'histoire constitutionnelle japonaise

\section{Chronique bibliographique}

Prix : $63 € \quad$ ISBN 978-2-275-02957-3

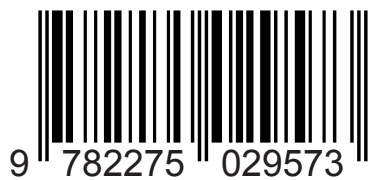

